\title{
The Stochastic Motion Roadmap: A Sampling Framework for Planning with Markov Motion Uncertainty
}

\author{
Ron Alterovitz \\ LAAS-CNRS, University of Toulouse \\ Toulouse, France \\ ron@ieor.berkeley.edu
}

\author{
Thierry Siméon \\ LAAS-CNRS, University of Toulouse \\ Toulouse, France \\ nic@laas.fr
}

\author{
Ken Goldberg \\ Departments of IEOR and EECS \\ University of California, Berkeley \\ goldberg@ieor.berkeley.edu
}

\begin{abstract}
We present a new motion planning framework that explicitly considers uncertainty in robot motion to maximize the probability of avoiding collisions and successfully reaching a goal. In many motion planning applications ranging from maneuvering vehicles over unfamiliar terrain to steering flexible medical needles through human tissue, the response of a robot to commanded actions cannot be precisely predicted. We propose to build a roadmap by sampling collision-free states in the configuration space and then locally sampling motions at each state to estimate state transition probabilities for each possible action. Given a query specifying initial and goal configurations, we use the roadmap to formulate a Markov Decision Process (MDP), which we solve using Infinite Horizon Dynamic Programming in polynomial time to compute stochastically optimal plans. The Stochastic Motion Roadmap (SMR) thus combines a sampling-based roadmap representation of the configuration space, as in PRM's, with the well-established theory of MDP's. Generating both states and transition probabilities by sampling is far more flexible than previous Markov motion planning approaches based on problem-specific or grid-based discretizations. We demonstrate the SMR framework by applying it to nonholonomic steerable needles, a new class of medical needles that follow curved paths through soft tissue, and confirm that SMR's generate motion plans with significantly higher probabilities of success compared to traditional shortest-path plans.
\end{abstract}

\section{INTRODUCTION}

In many applications of motion planning, the motion of the robot in response to commanded actions cannot be precisely predicted. Whether maneuvering a vehicle over unfamiliar terrain, steering a flexible needle through human tissue to deliver medical treatment, guiding a micro-scale swimming robot through turbulent water, or displaying a folding pathway of a protein polypeptide chain, the underlying motions cannot be predicted with certainty. But in many of these cases, a probabilistic distribution of feasible outcomes in response to commanded actions can be experimentally measured. This stochastic information is fundamentally different from a deterministic motion model. Though planning shortest feasible paths to the goal may be appropriate for problems with deterministic motion, shortest paths may be highly sensitive to uncertainties: the robot may deviate from its expected trajectory when moving through narrow passageways in the configuration space, resulting in collisions.

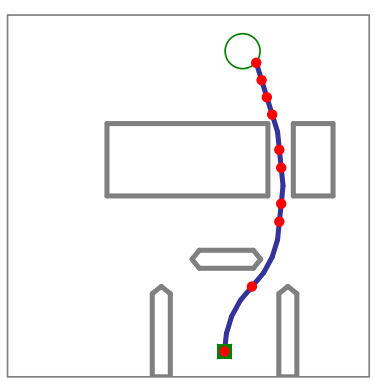

(a) Minimizing path length

$$
p_{s}=27 \%
$$

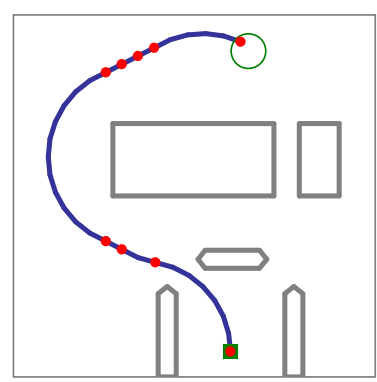

(b) Maximize probability of success using SMR $p_{s}=57 \%$
Fig. 1. The expected results of two plans to steer a Dubins-car mobile robot with left-right bang-bang steering and normally distributed motion uncertainty from an initial configuration (solid square) to a goal (open circle). Dots indicate steering direction changes. The Stochastic Motion Roadmap (SMR) introduces sampling of the configuration space and motion uncertainty model to generate plans that maximize the probability $p_{s}$ that the robot will successfully reach the goal without colliding with an obstacle. Evaluation of $p_{s}$ using multiple randomized simulations demonstrates that following a minimum length path under motion uncertainty (a) is substantially less likely to succeed than executing actions from an SMR plan (b).

In this paper, we develop a new motion planning framework that explicitly considers uncertainty in robot motion at the planning stage. Because future configurations cannot be predicted with certainty, we define a plan by actions that are a function of the robot's current configuration. A plan execution is successful if the robot does not collide with any obstacles and reaches the goal. The idea is to compute plans that maximize the probability of success.

Our framework builds on the highly successful approach used in Probabilistic Roadmaps (PRM's): a learning phase followed by a query phase [20]. During the learning phase, a random (or quasi-random) sample of discrete states is selected in the configuration space, and a roadmap is built that represents their collision-free connectivity. During the query phase, the user specifies initial and goal states, and the roadmap is used to find a feasible path that connects the initial state to the goal, possibly optimizing some criteria such as minimum length. PRM's have successfully solved many path planning problems for applications such as robotic manipulators and 
mobile robots $[12,22]$. The term "probabilistic" in PRM comes from the random sampling of states. An underlying assumption is that the collision-free connectivity of states is specified using boolean values rather than distributions.

In this paper, we relax this assumption and combine a roadmap representation of the configuration space with a stochastic model of robot motion. The input to our method is a geometric description of the workspace and a motion model for the robot capable of generating samples of the next configuration that the robot may attain given the current configuration and an action. We require that the motion model satisfy the Markovian property: the distribution of the next state depends only on the action and current state, which encodes all necessary past history. As in PRM's, the method first samples the configuration space, where the sampling can be random [20], pseudo-random [23], or utility-guided [11]. We then sample the robot's motion model to build a Stochastic Motion Roadmap (SMR), a set of weighted directed graphs with vertices as sampled states and edges encoding feasible state transitions and their associated probability of occurrence for each action.

The focus of our method is not to find a feasible motion plan, but rather to find an optimal plan that maximizes the probability that the robot will successfully reach a goal. Given a query specifying initial and goal configurations, we use the SMR to formulate a Markov Decision Process (MDP) where the "decision" corresponds to the action to be selected at each state in the roadmap. We solve the MDP in polynomial time using Infinite Horizon Dynamic Programming. Because the roadmap is a discrete representation of the continuous configuration space and transition probabilities, the computed optimal actions are approximations of the optimal actions in continuous space that converge as the roadmap increases in size. Although the plan, defined by the computed actions, is fixed, the path followed by the robot may differ each time the plan is executed because different state transitions may occur due to motion uncertainty. As shown in Fig. 1, plans that explicitly consider uncertainty to maximize the probability of success can differ substantially from traditional shortest path plans.

In SMR, "stochastic" refers to the motion of the robot, not to the sampling of states. PRM's were previously extended to explore stochastic motion in molecular conformation spaces $[4,5]$, but without a planning component to optimize actions. Our SMR formulation is applicable to a variety of decision-based robotics problems. It is particularly suited for nonholonomic robots, for which a deflection in the path due to motion uncertainty can result in failure to reach the goal, even if the deflection does not result in an immediate collision. The expansion of obstacles using their Minkowski sum [22] with a circle corresponding to an uncertainty tolerance is often sufficient for holonomic robots for which deflections can be immediately corrected, but this does not address collisions resulting from a nonholonomic constraint as in Fig. 2. By explicitly considering motion uncertainty in the planning phase, we hope to minimize such failures.

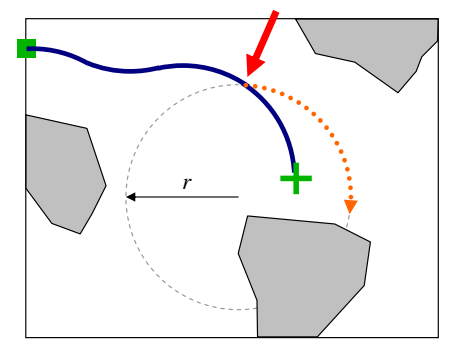

Fig. 2. The solid line path illustrates a motion plan from the start square to the goal (cross) for a nonholonomic mobile robot constrained to follow paths composed of continuously connected arcs of constant-magnitude curvature with radius of curvature $r$. If a deflection occurs at the location of the arrow, then the robot is unable to reach the goal due to the nonholonomic constraint, even if this deflection is immediately detected, since the robot cannot follow a path with smaller radius of curvature than the dotted line.

Although we use the terms robot and workspace, SMR's are applicable to any motion planning problem that can be modeled using a continuous configuration space and discrete action set with uncertain transitions between configurations. In this paper, we demonstrate a SMR planner using a variant of a Dubins car with bang-bang control, a nonholonomic mobile robot that can steer its wheels far left or far right while moving forward but cannot go straight. This model can generate motion plans for steerable needles, a new class of flexible bevel-tip medical needles that clinicians can steer through soft tissue around obstacles to reach targets inaccessible to traditional stiff needles $[2,36]$. As in many medical applications, considering uncertainty is crucial to the success of medical needle insertion procedures: the needle tip may deflect from the expected path due to tissue inhomogeneities that cannot be detected prior to the procedure. Due to uncertainty in predicted needle/tissue interactions, needle steering is illsuited to shortest-path plans that may guide the needle through narrow passageways between critical tissue such as blood vessels or nerves. By explicitly considering motion uncertainty using an SMR, we obtain solutions that result in possibly longer paths but that improve the probability of success.

\section{A. Related Work}

Motion planning can consider uncertainty in sensing (the current state of the robot and workspace is not known with certainty) and predictability (the future state of the robot and workspace cannot be deterministically predicted even when the current state and future actions are known) [22]. Extensive work has explored uncertainty associated with robot sensing, including SLAM and POMDP's to represent uncertainty in the current state $[33,12]$. In this paper, we assume the current state is known (or can be precisely determined from sensors), and we focus on the latter type of uncertainty, predictability.

Predictability can be affected by uncertainty in the workspace and by uncertainty in the robot's motion. Previous and ongoing work addresses many aspects of uncertainty in the workspace, including uncertainty in the goal location, such as in pursuit-evasion games [22, 24], and in dynamic environments with moving obstacles [35, 34, 27]. A recently 
developed method for grasp planning uses POMDP's to consider uncertainty in the configuration of the robot and the state of the objects in the world [18]. In this paper we focus explicitly on the case of uncertainty in the robot's motion rather than in goal or obstacle locations.

Apaydin et al. previously explored the connection between probabilistic roadmaps and stochastic motions using Stochastic Roadmap Simulation (SRS), a method designed specifically for molecular conformation spaces [4, 5]. SRS, which formalizes random walks in a roadmap as a Markov Chain, has been successfully applied to predict average behavior of molecular motion pathways of proteins and requires orders of magnitude less computation time than traditional MonteCarlo molecular simulations. However, SRS cannot be applied to more general robotics problems, including needle steering, because the probabilities associated with state transitions are specific to molecular scale motions and the method does not include a planning component to optimize actions.

Considering uncertainty in the robot's response to actions during planning results in a stochastic optimal control problem where feedback is generally required for success. Motion planners using grid-based numerical methods and geometric analysis have been applied to robots with motion uncertainty (sometimes combined with sensing uncertainty) using costbased objectives and worst-case analysis [26, 10, 25]. MDP's, a general approach that requires explicitly defining transition probabilities between states, have also been applied to motion planning by subdividing the workspace using regular grids and defining transition probabilities for motions between the grid cells $[14,16,22]$. These methods differ from SMR's since they use grids or problem-specific discretization.

Many existing planners for deterministic motion specialize in finding feasible paths through narrow passageways in complex configuration spaces using specialized sampling [3, 9] or learning approaches [29]. Since a narrow passageway is unlikely to be robust to motion uncertainty, finding these passageways is not the ultimate goal of our method. Our method builds a roadmap that samples the configuration space with the intent of capturing the uncertain motion transition probabilities necessary to compute optimal actions.

We apply SMR's to needle steering, a type of nonholonomic control-based motion planning problem. Nonholonomic motion planning has a long history in robotics and related fields [12, 22]. Past work has addressed deterministic curvature-constrained path planning with obstacles where a mobile robot's path is, like a car, constrained by a minimum turning radius [19, 21, 8, 31, 28]. For steerable needles, Park et al. applied a numeric diffusion-based method but did not consider obstacles or motion uncertainty [30]. Alterovitz et al. proposed an MDP formulation to find a stochastic shortest path for a steerable needle to a goal configuration, subject to userspecified "cost" parameters for direction changes, insertion distance, and collisions [2]. Because these costs are difficult to quantify, Alterovitz et al. introduced the objective function of maximizing probability of success [1]. These methods use a regular grid of states and an ad-hoc, identical discretization of the motion uncertainty distribution at all states. The methods do not consider the advantages of sampling states nor the use of sampling to estimate motion models.

\section{B. SMR Contributions}

SMR planning is a general framework that combines a roadmap representation of configuration space with the theory of MDP's to explicitly consider motion uncertainty at the planning stage to maximize the probability of success. SMR's use sampling to both learn the configuration space (represented as states) and to learn the stochastic motion model (represented as state transition probabilities). Sampling reduces the need for problem-specific geometric analysis or discretization for planning. As demonstrated by the success of PRM's, sampling states is useful for modeling complex configuration spaces that cannot be easily represented geometrically and extends well to higher dimensions. Random or quasi-random sampling reduces problems associated with regular grids of states, including the high computational complexity in higher dimensions and the sensitivity of solutions and runtimes to the selection of axes [22]. Sampling the stochastic motion model enables the use of a wide variety of motion uncertainty representations, including directly sampling experimentally measured data or using parameterized distributions such as a Gaussian distribution. This greatly improves previous Markov motion planning approaches that impose an ad-hoc, identical discretization of the transition probability distributions at all states [1].

Although SMR is a general framework, it provides improvements for steerable needle planning compared to previously developed approaches specifically designed for this application. Previous planners do not consider uncertainty in needle motion [30], or apply simplified models that only consider deflections at decision points and assume that all other motion model parameters are constant $[2,1]$. Because we use sampling to approximate the motion model rather than a problemspecific geometric approximation [2, 1], we eliminate the discretization error at the initial configuration and can easily include a more complex uncertainty model that considers arbitrary stochastic models for both insertion distance and radius of curvature. SMR's increase flexibility and decrease computation time; for problems with equal workspace size and expected values of motion model parameters, a query that requires over a minute to solve using a grid-based MDP due to the large number of states needed to bound discretization error [1] requires just 6 seconds using an SMR.

\section{Algorithm}

\section{A. Input}

To build an SMR, the user must first provide input parameters and function implementations to describe the configuration space and robot motion model. A configuration of the robot and workspace is defined by a vector $x \in C=$ $\Re^{d}$, where $d$ is the number of degrees of freedom in the configuration space $C$. At any configuration $x$, the robot can perform an action from a discrete action set $U$ of size $w$. The bounds of the configuration space are defined by $B_{i}^{\text {min }}$ 
and $B_{i}^{\max }$ for $i=1, \ldots, d$, which specify the minimum and maximum values, respectively, for each configuration degree of freedom $i$. The functions isCollisionFree $(x)$ and isCollisionFreePath $(x, y)$ implicitly define obstacles within the workspace; the former returns true if configuration $x$ collides with an obstacle and false otherwise, and the latter returns true if the path (computed by a local planner [20]) from configuration $x$ to $y$ collides with an obstacle and false otherwise. (We consider exiting the workspace as equivalent to colliding with an obstacle.) The function distance $(x, y)$ specifies the distance between two configurations $x$ and $y$, which can equal the Euclidean distance in $d$-dimensional space or some other user-specified distance metric. The function generateSampleTransition $(x, u)$ implicitly defines the motion model and its probabilistic nature; this function returns a sample from a known probability distribution for the next configuration given that the robot is currently in configuration $x$ and will perform action $u$.

\section{B. Building the Roadmap}

We build the stochastic motion roadmap using the algorithm buildSMR defined in Procedure 1. The roadmap is defined by a set of vertices $V$ and sets of edges $E^{u}$ for each action $u \in U$. The algorithm first samples $n$ collision-free states in the configuration space and stores them in $V$. In our implementation, we use a uniform random sampling of the configuration space inside the bounds defined by $\left(B_{i}^{\min }, B_{i}^{\max }\right)$ for $i=1, \ldots, d$, although other random distributions or quasi- random sampling methods could be used $[12,23]$. For each state $s \in V$ and an action $u \in U$, buildSMR calls the function getTransitions, defined in Procedure 2, to obtain a set of possible next states in $V$ and probabilities of entering those states when action $u$ is performed. We use this set to add to $E^{u}$ weighted directed edges $(s, t, p)$, which specify the probability $p$ that the robot will transition from state $s \in V$ to state $t \in V$ when currently in state $s$ and executing action $u$.

The function getTransitions, defined in Procedure 2, estimates state transition probabilities. Given the current state $s$ and an action $u$, it calls the problem-specific function generateSampleTransition $(x, u)$ to generate a sample configuration $q$ and then selects the state $t \in V$ closest to $q$ using the problem-specific distance function. We repeat this motion sampling $m$ times and then estimate the probability of transitioning from state $s$ to $t$ as the proportion of times that this transition occurred out of the $m$ samples. If there is a collision in the transition from state $s$ to $t$, then the transition is replaced with a transition from $s$ to a dedicated "obstacle state," which is required to estimate the probability that the robot collides with an obstacle.

This algorithm has the useful property that the transition probability from state $s$ to state $t$ in the roadmap equals the fraction of transition samples that fall inside state $t$ 's Voronoi cell. This property is implied by the use of nearest neighbor checking in getTransitions. As $m \rightarrow \infty$, the probability $p$ of transitioning from $s$ to $t$ will approach, with probability 1 , the integral of the true transition distribution over the Voronoi

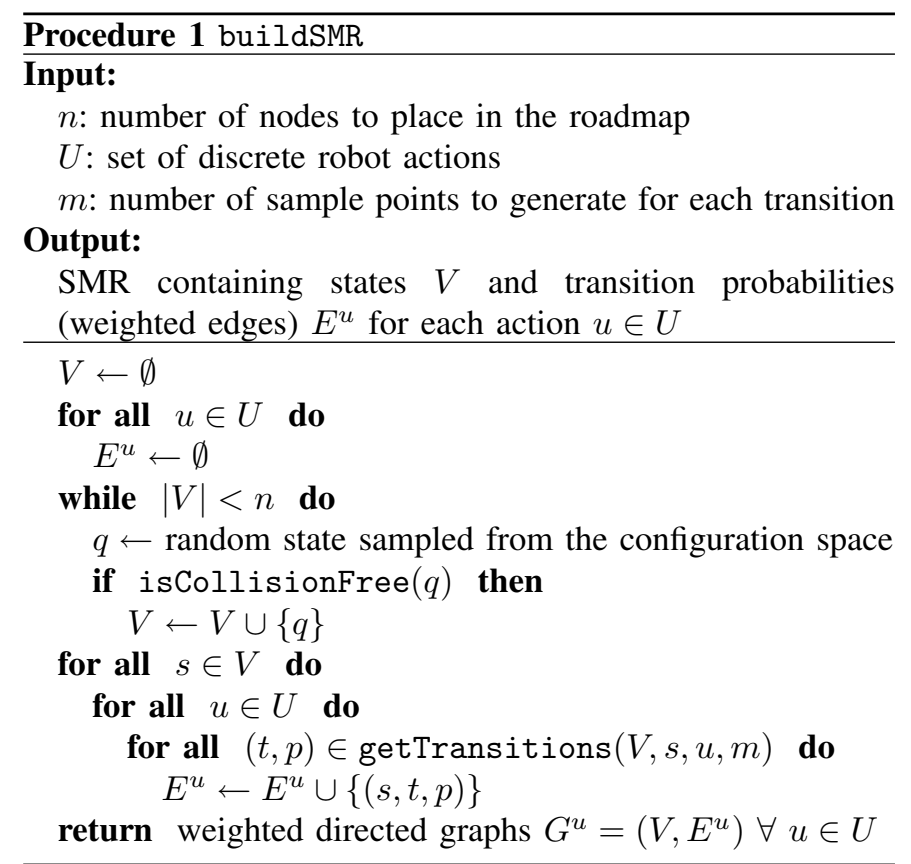

cell of $t$. As the number of states $n \rightarrow \infty$, the expected volume $V_{t}$ of the Voronoi cell for state $t$ equals $V / n \rightarrow 0$, where $V$ is the volume of the configuration space. Hence, the error in the approximation of the probability $p$ due to the use of a discrete roadmap will decrease as $n$ and $m$ increase.

\section{Solving a Query}

We define a query by an initial configuration $s^{*}$ and a set of goal configurations $T^{*}$.

Using the SMR and the query input, we build an $n \times n$ transition probability matrix $P(u)$ for each $u \in U$. For each tuple $(s, t, p) \in E^{u}$, we set $P_{s t}(u)=p$ so $P_{s t}(u)$ equals the probability of transitioning from state $s$ to state $t$ given that action $u$ is performed. We store each matrix $P(u)$ as a sparse matrix that only includes pointers to a list of non-zero elements in each row and assume all other entries are 0.

We define $p_{s}(i)$ to be the probability of success given that the robot is currently in state $i$. If the position of state $i$ is inside the goal, $p_{s}(i)=1$. If the position of state $i$ is inside an obstacle, $p_{s}(i)=0$. Given an action $u_{i}$ for some other state $i$, the probability of success will depend on the response of the robot to the action and the probability of success from the next state. The goal of our motion planner is to compute an optimal action $u_{i}$ to maximize the expected probability of success at every state $i$ :

$$
p_{s}(i)=\max _{u_{i}}\left\{E\left[p_{s}(j) \mid i, u_{i}\right]\right\},
$$

where the expectation is over $j$, a random variable for the next state. Since the roadmap is a discrete approximation of the continuous configuration space, we expand the expected value in Eq. 1 to a summation:

$$
p_{s}(i)=\max _{u_{i}}\left\{\sum_{j \in V} P_{i j}\left(u_{i}\right) p_{s}(j)\right\} .
$$




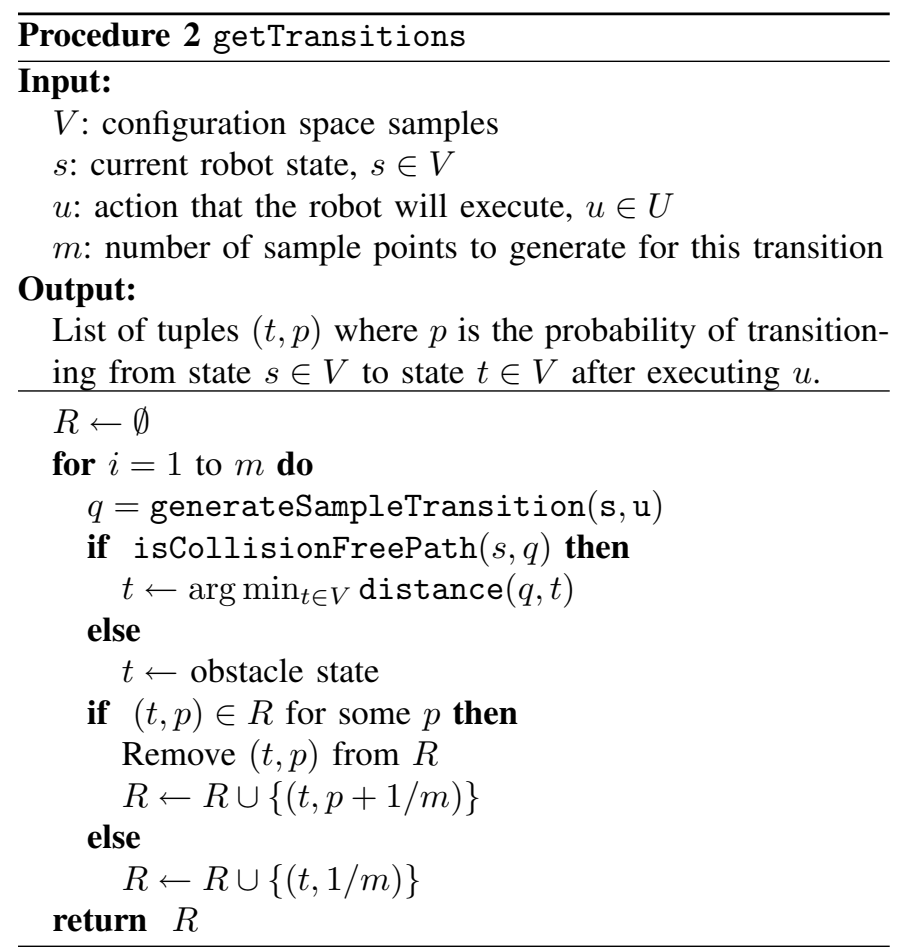

We observe that Eq. 2 is an MDP and has the form of the Bellman equation for a stochastic shortest path problem [7]:

$$
J^{*}(i)=\max _{u_{i}} \sum_{j \in V} P_{i j}\left(u_{i}\right)\left(g\left(i, u_{i}, j\right)+J^{*}(j)\right) .
$$

where $g\left(i, u_{i}, j\right)$ is a "reward" for transitioning from state $i$ to $j$ after action $u_{i}$. In our case, $g\left(i, u_{i}, j\right)=0$ for all $i, u_{i}$, and $j$, and $J^{*}(i)=p_{s}(i)$.

Stochastic shortest path problems of the form in Eq. 3 can be optimally solved using infinite horizon dynamic programming. For stationary Markovian problems, the configuration space does not change over time, which implies that the optimal action at each state is purely a function of the state without explicit dependence on time. Infinite horizon dynamic programming is a type of dynamic programming (DP) in which there is no finite time horizon [7]. Specifically, we use the value iteration algorithm [7], which iteratively updates $p_{s}(i)$ for each state $i$ by evaluating Eq. 3. This generates a DP lookup table containing the optimal action $u_{i}$ and the probability of success $p_{s}(i)$ for each $i \in V$.

The algorithm is guaranteed to terminate in $n$ (the number of states) iterations if the transition probability graph corresponding to some optimal stationary policy is acyclic [7]. Violation of this requirement can occur in rare cases in which a cycle is feasible and deviating from the cycle will result in imminent failure. To remove this possibility, we introduce a small penalty $\gamma$ for each transition by setting $g\left(i, u_{i}, j\right)=-\gamma$ in Eq. 3. Increasing $\gamma$ has the effect of giving preference to shorter paths at the expense of a less precise estimate of the probability of success, where the magnitude of the error is (weakly) bounded by $\gamma n$.

\section{Computational Complexity}

Building an SMR requires $O(n)$ time to create the states $V$, not including collision detection. Generating the edges in $E^{u}$ requires $O(w n)$ calls to generateTransitions, where $w=$ $|U|$. For computational efficiency, it is not necessary to consolidate multiple tuples with the same next state $t$; the addition $p+1 / m$ can be computed automatically during value iteration. Hence, each call requires $O(m d n)$ time using brute-force nearest neighbor checking. For certain low-dimensional configuration spaces, this can be reduced to $O(m \exp (d) \log (n))$ using kd-trees [6]. Hence, the total time complexity of building an SMR is $O\left(w m d n^{2}\right)$ or $O(w m \exp (d) n \log (n))$. This does not include the cost of $n$ state collision checks and $n m$ checks of collision free paths, which are problem-specific and may increase the computational complexity depending on the workspace definition.

Solving a query requires building the transition probability matrices and executing value iteration. Although the matrices $P_{i j}(u)$ each have $n^{2}$ entries, we do not store the zero entries as described above. Since the robot will generally only transition to a state $j$ in the spatial vicinity of state $i$, each row of $P_{i j}(u)$ has only $k$ nonzero entries, where $k<<n$. Building the sparse matrices requires $O(w k n)$ time. By only accessing the nonzero entries of $P_{i j}(u)$ during the value iteration algorithm, each iteration for solving a query requires only $O(w k n)$ rather than $O\left(w n^{2}\right)$ time. Thus, the value iteration algorithm's total time complexity is $O\left(w k n^{2}\right)$ since the number of iterations is bounded by $n$. To further improve performance, we terminate value iteration when the maximum change $\epsilon$ over all states is less than some user-specified threshold $\epsilon^{*}$. In our test cases, we used $\epsilon^{*}=10^{-7}$, which resulted in far fewer than $n$ iterations.

\section{SMR fOR Medical NeEdle SteERING}

Diagnostic and therapeutic medical procedures such as extracting tissue samples for biopsies, injecting drugs for anesthesia, or implanting radioactive seeds for brachytherapy cancer treatment require insertion of a needle to a specific location in soft tissue. A new class of needles, steerable needles, are composed of a flexible material and with a bevel-tip that can be steered to targets in soft tissue that are inaccessible to traditional stiff needles [36, 37, 2]. Steerable needles are controlled by two degrees of freedom actuated at the needle base: insertion distance and bevel direction. When the needle is inserted, the asymmetric force applied by the bevel causes the needle to bend and follow a path of constant curvature through the tissue in the direction of the bevel [36]. Webster et al. experimentally demonstrated that, under ideal conditions, the curve has a constant radius of curvature $r$.

We assume the workspace is extracted from a medical image, where obstacles represent tissues that cannot be cut by the needle, such as bone, or sensitive tissues that should not be damaged, such as nerves or arteries. In this paper we consider motion plans in an imaging plane since the speed/resolution trade-off of 3-D imaging modalities is generally poor for 3D interventional applications. We assume the needle moves a distance $\delta$ between image acquisitions that are used to 


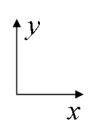

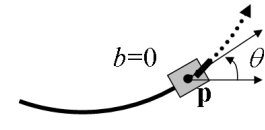

(a) (b)

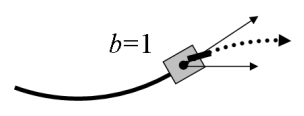

Fig. 3. The state of a bang-bang steering car is defined by point $\mathbf{p}$, orientation $\theta$, and turning direction $b$ (a). The car moves forward along an arc of constant curvature and can turn either left (a) or right (b).

determine the current needle position and orientation. We do not consider motion by the needle out of the imaging plane or needle retraction, which modifies the tissue and can influence future insertions. When restricted to motion in a plane, the bevel direction can be set to point left $(b=0)$ or right $(b=1)$ [36]. Due to the nonholonomic constraint imposed by the bevel, the motion of the needle tip can be modeled as a bangbang steering car, a variant of a Dubins car that can only turn its wheels far left or far right while moving forward $[36,1]$.

Clinicians performing medical needle insertion procedures must consider uncertainty in the needle's motion through tissue due to patient differences and the difficulty in predicting needle/tissue interaction. Bevel direction changes further increase uncertainty due to stiffness along the needle shaft. Medical imaging in the operating room can be used to measure the needle's current position and orientation to provide feedback to the planner $[13,15]$, but this measurement by itself provides no information about the effect of future deflections during insertion due to motion uncertainty.

Stochastic motion roadmaps offer features particularly beneficial for medical needle steering. First, SMR's explicitly consider uncertainty in the motion of the needle. Second, intraoperative medical imaging can be combined with the fast SMR queries to permit control of the needle in the operating room without requiring time-consuming intra-operative re-planning.

\section{A. SMR Implementation}

We formulate the SMR for a bang-bang steering car, which can be applied to needle steering. The state of such a car is fully characterized by its position $\mathbf{p}=(x, y)$, orientation angle $\theta$, and turning direction $b$, where $b$ is either left $(b=0)$ or right $(b=1)$. Hence, the dimension of the state space is $d=4$, and a state $i$ is defined by $s_{i}=\left(x_{i}, y_{i}, \theta_{i}, b_{i}\right)$, as illustrated in Fig. 3. We encode $b_{i}$ in the state since it is a type of history parameter that is required by the motion uncertainty model. Since an SMR assumes that each component of the state vector is a real number, we define the binary $b_{i}$ as the floor of the fourth component in $s_{i}$, which we bound in the range $[0,2)$.

Between sensor measurements of state, we assume the car moves a distance $\delta$. The set $U$ consists of two actions: move forward turning left $(u=0)$, or move forward turning right $(u=1)$. As the car moves forward, it traces an arc of length $\delta$ with radius of curvature $r$ and direction based on $u$. We consider $r$ and $\delta$ as random variables drawn from a given distribution. In this paper, we consider $\delta \sim N\left(\delta_{0}, \sigma_{\delta_{a}}\right)$ and $r \sim$ $N\left(r_{0}, \sigma_{r_{a}}\right)$, where $N$ is a normal distribution with given mean and standard deviation parameters and $a \in\{0,1\}$ indicates di-

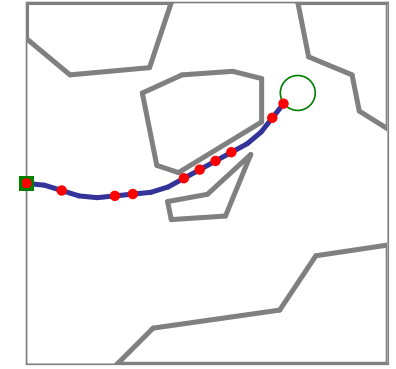

(a) Minimizing path length $p_{s}=35 \%$

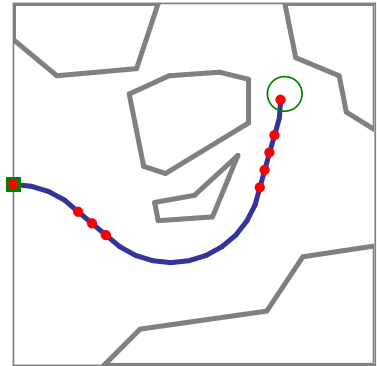

(b) Maximizing $p_{s}$ using SMR $p_{s}=83 \%$
Fig. 4. Explicitly considering motion uncertainty using an SMR planner improves the probability of success.

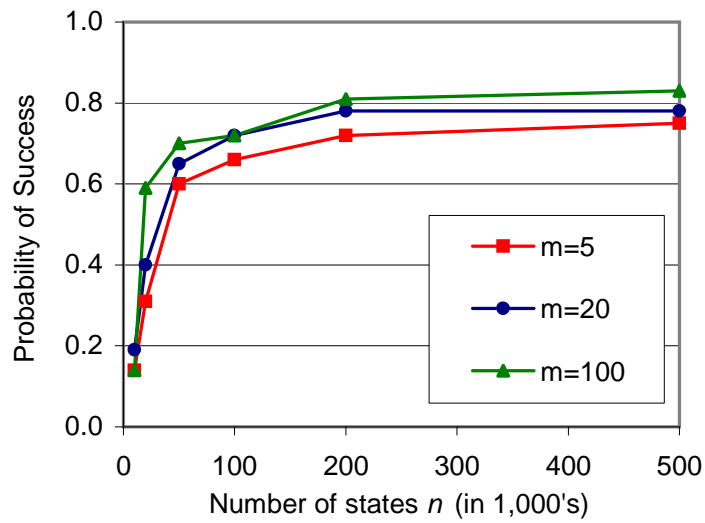

Fig. 5. Effect of the number of states $n$ and number of motion samples $m$ on the probability of success $p_{s}$.

rection change. We implement generateSampleTransition to draw random samples from these distributions. Although the uncertainty parameters can be difficult to measure precisely, even rough estimates may be more realistic than using deterministic transitions when uncertainty is high.

We define the workspace as a rectangle of width $x_{\max }$ and height $y_{\max }$ and define obstacles as polygons in the plane. To detect obstacle collisions, we use the zerowinding rule [17]. We define the distance between two states $s_{1}$ and $s_{2}$ to be the weighted Euclidean distance between the poses plus an indicator variable to ensure the turning directions match: distance $\left(s_{1}, s_{2}\right)=$ $\sqrt{\left(x_{1}-x_{2}\right)^{2}+\left(y_{1}-y_{2}\right)^{2}+\alpha\left(\theta_{1}-\theta_{2}\right)^{2}}+M$, where $M \rightarrow$ $\infty$ if $b_{1} \neq b_{2}$, and $M=0$ otherwise. For fast nearest-neighbor computation, we use the CGAL implementation of kd-trees [32]. Since the distance function is non-Euclidean, we use the formulation developed by Atramentov and LaValle to build the kd-tree [6]. We define the goal $T^{*}$ as all configuration states within a ball of radius $t^{r}$ centered at a point $\mathbf{t}^{*}$.

\section{B. Results}

We implemented the SMR planner in $\mathrm{C}++$ and tested the method on workspaces of size $x_{\max }=y_{\max }=10$ with polygonal obstacles as shown in shown in Fig. 1 and Fig. 4. We set the robot parameters $r_{0}=2.5$ and $\delta_{0}=0.5$ with motion 
uncertainty parameters $\sigma_{\delta_{0}}=0.1, \sigma_{\delta_{1}}=0.2, \sigma_{r_{0}}=0.5$, and $\sigma_{r_{1}}=1.0$. We set parameters $\gamma=0.00001$ and $\alpha=2.0$. We tested the motion planner on a $2.2 \mathrm{GHz}$ AMD Opteron PC. Building the SMR required approximately 1 minute for $n=50,000$ states, executing a query required 6 seconds, and additional queries for the same goal required less than 1 second of computation time for both example problems.

We evaluate the plans generated by SMR with multiple randomized simulations. Given the current state of the robot, we query the SMR to obtain an optimal action $u$. We then execute this action and compute the expected next state. We repeat until the robot reaches the goal or hits an obstacle, and we illustrate the resulting expected path. Since the motion response of the robot to actions is not deterministic, success of the procedure can rarely be guaranteed. To estimate $p_{s}$, we run the simulation 100 times, sampling the next state from the transition probability distribution rather than selecting the expected value, and we compute the number of goal acquisitions divided by the number of obstacle collisions.

In Fig. 4(b), we illustrate the expected path using an SMR with $m=100$ motion samples and $n=500,000$ states. As in Fig. 1(b), the robot avoids passing through a narrow passageway near the goal and instead takes a longer route. The selection of the longer path is not due to insufficient states in the SMR; there exist paths in the SMR that pass through the narrow gaps between the obstacles. The plan resulting in a longer path is selected purely because it maximizes $p_{s}$.

The probability of success $p_{s}$ improves as the sampling density of the configuration space and the motion uncertainty distribution increase, as shown in Fig. 5. As $n$ and $m$ increase, $p_{s}(s)$ is more accurately approximated over the configuration space, resulting in better action decisions. However, $p_{s}$ effectively converges for $n \geq 100,000$ and $m \geq 20$, suggesting the inherent difficulty of the motion planning problem. Furthermore, the expected path does not substantially vary from the path shown in Fig. 4(b) for $n \geq 50,000$ and $m \geq 5$. The number of states required by the SMR planner is far smaller than the 800,000 states required for a similar problem using a grid-based approach with bounded error [1].

In Fig. 4(a), we computed the optimal shortest path assuming deterministic motion of the robot using a fine regular discrete grid with 816,080 states for which the error due to discretization is small and bounded [1]. We estimate $p_{s}$ using the same simulation methodology as for an SMR plan, except that we compute the shortest path for each query. The expected shortest path passes through a narrow passage between obstacles and the resulting probability of success is substantially lower compared to the SMR plan. The result was similar for the example in Fig. 1; explicitly considering motion uncertainty improved the probability of success.

To further illustrate the importance of explicitly considering uncertainty during motion planning, we vary the standard deviation parameters $\sigma_{\delta_{0}}, \sigma_{\delta_{1}}, \sigma_{r_{0}}$, and $\sigma_{r_{1}}$. In Fig. 6, we compute a plan for a robot with each standard deviation parameter set to a quarter of its default value. For this low uncertainty case, the uncertainty is not sufficient to justify

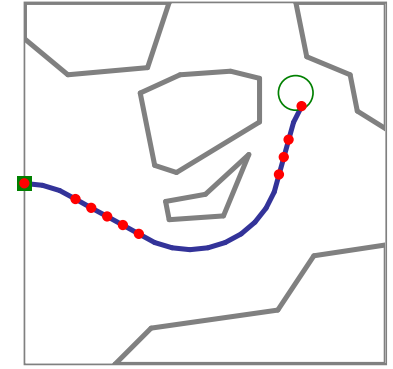

(a) High uncertainty $p_{s}=78 \%$

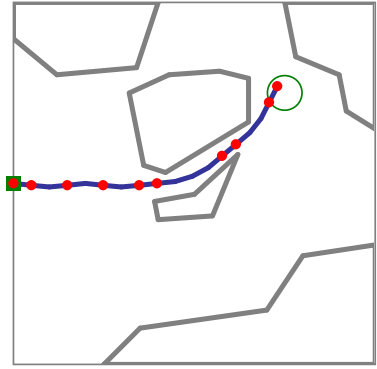

(b) Low uncertainty $p_{s}=87 \%$
Fig. 6. The level of uncertainty affects SMR planning results. In cases of low uncertainty (with $75 \%$ reduction in distribution standard deviations), the expected path resembles a deterministic shortest path due to the small influence of uncertainty on $p_{s}$ and the effect of the penalty term $\gamma$. In both these examples, the same $n=200,000$ states were used in the roadmap.

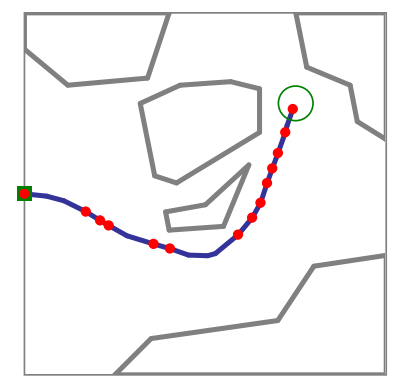

(a) Successful procedure

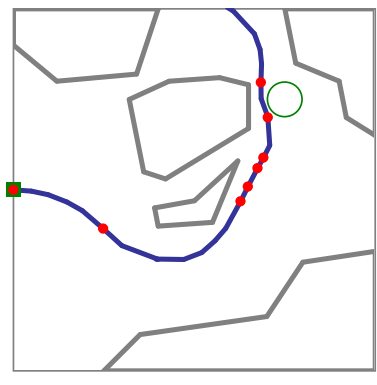

(b) Unsuccessful procedure
Fig. 7. Two simulated procedures of needle steering, one successful (a) and one unsuccessful due to effects of uncertain motion (b), using an SMR with $n=50,000$ states

avoiding the narrow passageway; the penalty $\gamma$ causes the plan to resemble the deterministic shortest plan in Fig. 4(a). Also, $p_{s}$ is substantially higher because of the lower uncertainty.

In Fig. 7, we execute the planner in the context of an image-guided procedure. We assume the needle tip position and orientation is extracted from a medical image and then execute a query, simulate the needle motion by drawing a sample from the motion uncertainty distribution, and repeat. The effect of uncertainty can be seen as deflections in the path. In practice, clinicians could monitor $p_{s}(s)$ for the current state $s$ as the procedure progresses.

\section{CONCLUSION}

In many motion planning applications, the response of the robot to commanded actions cannot be precisely predicted. We introduce the Stochastic Motion Roadmap (SMR), a new motion planning framework that explicitly considers uncertainty in robot motion to maximize the probability that a robot will avoid obstacle collisions and successfully reach a goal. SMR planners combine the roadmap representation of configuration space used in PRM with the theory of MDP's to explicitly consider motion uncertainty at the planning stage.

To demonstrate SMR's, we considered a nonholonomic mobile robot with bang-bang control, a type of Dubins-car robot model that can be applied to steering medical needles 
through soft tissue. Needle steering, like many other medical procedures, is subject to substantial motion uncertainty and is therefore ill-suited to shortest-path plans that may guide medical tools through narrow passageways between critical tissues. Using randomized simulation, we demonstrated that SMR's generate motion plans with significantly higher probabilities of success compared to traditional shortest-path approaches.

In future work, we will extend the SMR framework and explore new applications with higher dimensional configuration spaces. We plan to extend the formulation to also consider actions in a continuous range rather than solely from a discrete set, to investigate more sophisticated sampling methods for generating configuration samples and for estimating transition probabilities, and to integrate the effects of sensing uncertainty. We also plan to explore new biomedical and industrial applications where uncertainty in motion should be considered to maximize the probability of success.

\section{ACKNOWLEDGMENT}

This work has been partially supported by the ITAV project ALMA.

\section{REFERENCES}

[1] R. Alterovitz, M. Branicky, and K. Goldberg, "Constant-curvature motion planning under uncertainty with applications in image-guided medical needle steering," in Proc. Int. Workshop on the Algorithmic Foundations of Robotics, July 2006.

[2] R. Alterovitz, A. Lim, K. Goldberg, G. S. Chirikjian, and A. M Okamura, "Steering flexible needles under Markov motion uncertainty," in Proc. IEEE/RSJ Int. Conf. on Intelligent Robots and Systems (IROS), Aug. 2005, pp. 120-125.

[3] N. M. Amato, O. B. Bayazit, L. K. Dale, C. Jones, and D. Vallejo, "OBPRM: An obstacle-based PRM for 3D workspaces," in Robotics: The Algorithmic Perspective, P. Agarwal, L. E. Kavraki, and M. Mason, Eds. Natick, MA: AK Peters, Ltd., 1998, pp. 156-168.

[4] M. S. Apaydin, D. L. Brutlag, C. Guestrin, D. Hsu, and J.-C. Latombe, "Stochastic roadmap simulation: an efficient representation and algorithm for analyzing molecular motion," in Proc. RECOMB, 2002, pp. $12-21$.

[5] M. Apaydin, D. Brutlag, C. Guestrin, D. Hsu, and J.-C. Latombe, "Stochastic conformational roadmaps for computing ensemble properties of molecular motion," in Algorithmic Foundations of Robotics V. Berlin, Germany: Springer-Verlag, 2003, pp. 131-148.

[6] A. Atramentov and S. M. LaValle, "Efficient nearest neighbor searching for motion planning," in Proc. IEEE Int. Conf. on Robotics and Automation (ICRA), 2002.

[7] D. P. Bertsekas, Dynamic Programming and Optimal Control, 2nd ed. Belmont, MA: Athena Scientific, 2000.

[8] A. Bicchi, G. Casalino, and C. Santilli, "Planning shortest boundedcurvature paths for a class of nonholonomic vehicles among obstacles," Journal of Intelligent and Robotic Systems, vol. 16, pp. 387-405, 1996.

[9] V. Boor, N. H. Overmars, and A. F. van der Stappen, "The Gaussian sampling strategy for probabilisitic roadmap planners," in Proc. IEEE Int. Conf. on Robotics and Automation (ICRA), 1999, pp. 1018-1023.

[10] B. Bouilly, T. Siméon, and R. Alami, "A numerical technique for planning motion strategies of a mobile robot in presence of uncertainty," in Proc. IEEE Int. Conf. on Robotics and Automation (ICRA), Nagoya, Japan, May 1995.

[11] B. Burns and O. Brock, "Toward optimal configuration space sampling," in Proc. Robotics: Science and Systems, Cambridge, MA, June 2005.

[12] H. Choset, K. M. Lynch, S. Hutchinson, G. Kantor, W. Burgard, L. E. Kavraki, and S. Thrun, Principles of Robot Motion: Theory, Algorithms, and Implementations. MIT Press, 2005.

[13] K. Cleary, L. Ibanez, N. Navab, D. Stoianovici, A. Patriciu, and G. Corral, "Segmentation of surgical needles for fluoroscopy servoing using the Insight Software Toolkit (ITK)," in Proc. Int. Conf. of the IEEE Engineering In Medicine and Biology Society, Sept. 2003, pp. 698-701.
[14] T. Dean, L. P. Kaelbling, J. Kirman, and A. Nicholson, "Planning under time constraints in stochastic domains," Artificial Intelligence, vol. 76, no. 1-2, pp. 35-74, July 1995.

[15] S. P. DiMaio, D. F. Kacher, R. E. Ellis, G. Fichtinger, N. Hata, G. P. Zientara, L. P. Panych, R. Kikinis, and F. A. Jolesz, "Needle artifact localization in 3T MR images," in Medicine Meets Virtual Reality 14, J. D. Westwood et al., Eds. IOS Press, Jan. 2006, pp. 120-125.

[16] D. Ferguson and A. Stentz, "Focussed dynamic programming: Extensive comparative results," Robotics Institute, Carnegie Mellon University, Pittsburgh, PA, Tech. Rep. CMU-RI-TR-04-13, Mar. 2004.

[17] D. Hearn and M. P. Baker, Computer Graphics with OpenGL, 3rd ed. Prentice Hall, 2003.

[18] K. Hsiao, L. P. Kaelbling, and T. Lozano-Perez, "Grasping POMDPs," in Proc. IEEE Int. Conf. on Robotics and Automation (ICRA), Rome, Italy, Apr. 2007, pp. 4685-4692.

[19] P. Jacobs and J. Canny, "Planning smooth paths for mobile robots," in Proc. IEEE Int. Conf. on Robotics and Automation (ICRA), May 1989, pp. 2-7.

[20] L. E. Kavraki, P. Svestka, J.-C. Latombe, and M. Overmars, "Probabilistic roadmaps for path planning in high dimensional configuration spaces," IEEE Trans. on Robotics and Automation, vol. 12, no. 4, pp. 566-580, 1996

[21] J.-P. Laumond, P. E. Jacobs, M. Taïx, and R. M. Murray, "A motion planner for nonholonomic mobile robots," IEEE Trans. on Robotics and Automation, vol. 10, no. 5, pp. 577 - 593, Oct. 1994.

[22] S. M. LaValle, Planning Algorithms. Cambridge, U.K.: Cambridge University Press, 2006.

[23] S. M. LaValle, M. S. Branicky, and S. R. Lindemann, "On the relationship between classical grid search and probabilistic roadmaps," Int. J. of Robotics Research, vol. 23, no. 7/8, pp. 673-692, July 2004.

[24] S. M. LaValle, D. Lin, L. J. Guibas, J.-C. Latombe, and R. Motwani, "Finding an unpredictable target in a workspace with obstacles," in Proc. IEEE Int. Conf. on Robotics and Automation (ICRA), 1997, pp. 737-742.

[25] S. M. LaValle and S. A. Hutchinson, "An objective-based framework for motion planning under sensing and control uncertainties," Int. J. of Robotics Research, vol. 17, no. 1, pp. 19-42, Jan. 1998.

[26] A. Lazanas and J. Latombe, "Motion planning with uncertainty: A landmark approach," Artificial Intelligence, vol. 76, no. 1-2, pp. 285317, 1995.

[27] S. R. Lindemann, I. I. Hussein, and S. M. LaValle, "Real time feedback control for nonholonomic mobile robots with obstacles," in Proc. IEEE Conf. Decision and Control, 2006.

[28] R. Mason and J. Burdick, "Trajectory planning using reachable-state density functions," in Proc. IEEE Int. Conf. on Robotics and Automation (ICRA), vol. 1, May 2002, pp. 273-280.

[29] A. W. Moore and C. G. Atkeson, "The parti-game algorithm for variable resolution reinforcement learning in multidimensional state spaces," Machine Learning, vol. 21, no. 3, pp. 199-233, 1995.

[30] W. Park, J. S. Kim, Y. Zhou, N. J. Cowan, A. M. Okamura, and G. S. Chirikjian, "Diffusion-based motion planning for a nonholonomic flexible needle model," in Proc. IEEE Int. Conf. on Robotics and Automation (ICRA), Apr. 2005, pp. 4611-4616.

[31] J. Sellen, "Approximation and decision algorithms for curvatureconstrained path planning: A state-space approach," in Robotics: The Algorithmic Perspective, P. K. Agarwal, L. E. Kavraki, and M. T. Mason, Eds. Natick, MA: AK Peters, Ltd., 1998, pp. 59-67.

[32] H. Tangelder and A. Fabri, "dD spatial searching," in CGAL-3.2 User and Reference Manual, CGAL Editorial Board, Ed., 2006. [Online]. Available: http://www.cgal.org/Manual/

[33] S. Thrun, W. Burgard, and D. Fox, Probabilistic Robotics. MIT Press, 2005.

[34] J. van den Berg and M. Overmars, "Planning the shortest safe path amidst unpredictably moving obstacles," in Proc. Int. Workshop on the Algorithmic Foundations of Robotics, July 2006.

[35] D. Vasquez, F. Large, T. Fraichard, and C. Laugier, "High-speed autonomous navigation with motion prediction for unknown moving obstacles," in Proc. IEEE/RSJ Int. Conf. on Intelligent Robots and Systems (IROS), 2004, pp. 82-87.

[36] R. J. Webster III, J. S. Kim, N. J. Cowan, G. S. Chirikjian, and A. M. Okamura, "Nonholonomic modeling of needle steering," Int. $J$ of Robotics Research, vol. 25, pp. 509-525, May 2006.

[37] R. J. Webster III, J. Memisevic, and A. M. Okamura, "Design considerations for robotic needle steering," in Proc. IEEE Int. Conf. on Robotics and Automation (ICRA), Apr. 2005, pp. 3599-3605. 